\title{
Determinación de la seguridad de la inmunoterapia subcutánea con extractos polimerizados de Dermatophagoides farinae, Dermatophagoides pteronyssinus y Blomia tropicalis administrada en pacientes con enfermedad alérgica
}

\author{
Safety determination of subcutaneous immunotherapy with \\ Dermatophagoides farinae, Dermatophagoides pteronyssinus and Blomia \\ tropicalis polymerized extracts administered in allergic disease patients
}

Iris Y. Castelblanco-Arango $M D^{1}$, Paola A. Gómez-Pineda MD², Ricardo Cardona-Villa MSc ${ }^{3}$

Introducción: las enfermedades alérgicas afectan la calidad de vida de quienes las padecen y en los últimos años su prevalencia ha aumentado significativamente. La inmunoterapia con extractos alergénicos es usada para mejorar el curso de la enfermedad; no obstante, aún es necesario conocer su efectividad y seguridad. Objetivo: describir las reacciones adversas sistémicas producidas por la administración de inmunoterapia subcutánea con extractos polimerizados de ácaros. Materiales y métodos: se realizó un estudio de cohorte retrospectivo de pacientes con enfermedad alérgica mediada por IgE que recibieron inmunoterapia subcutánea con extractos polimerizados de Dermatophagoides farinae, Dermatophagoides pteronyssinus y Blomia tropicalis en el servicio de Alergología Clínica de la IPS Universitaria de la Universidad de Antioquia (Medellín, Colombia). Se realizó un

\footnotetext{
${ }^{1}$ Médica y cirujana, especialista en Alergología Clínica. IPS Universitaria, Grupo de Alergología Clínica y Experimental (GACE), Facultad de Medicina, Universidad de Antioquia. Medellín, Colombia. Correo electrónico: irisyan35@hotmail.com

2 Médica y cirujana, especialista en Alergología Clínica. Grupo de Alergología Clínica y Experimental (GACE), Facultad de Medicina, Universidad de Antioquia. Medellín, Colombia.

${ }_{3}^{3}$ Médico y cirujano, especialista en Alergología Clínica, MSc en Inmunología. Docente e investigador, Grupo de Alergología Clínica y Experimental (GACE), Facultad de Medicina, Universidad de Antioquia. Medellín, Colombia.

Conflicto de intereses: los autores declaran que no tienen conflicto de intereses Medicina \& Laboratorio 2016; 22: 365-380

Módulo 19 (Investigación), número 47. Editora Médica Colombiana S.A. $2016^{\circ}$

Recibido el 10 de agosto de 2016; aceptado el 30 de agosto de 2016
} 
análisis descriptivo de las variables demográficas y clínicas y su asociación con el riesgo de reacción adversa sistémica. Resultados: se incluyeron 622 pacientes; $92,8 \%$ con diagnóstico de rinitis, $60,3 \%$ de asma, 56,9\% de conjuntivitis y $19,6 \%$ de dermatitis atópica. Un total de 73 (11,7\%) pacientes experimentaron reacciones sistémicas adversas (razón de reacción/inyección $=1,06 \times 100$ inyecciones); de estos 47 (64,4\%) recibieron adrenalina intramuscular. El 93,1\% de los pacientes presentaron reacciones sistémicas grado 1 y 2 . El asma fue el diagnóstico con mayor riesgo de presentar reacción sistémica (riesgo relativo $=1,96$ ). Conclusiones: la inmunoterapia subcutánea con extractos polimerizados de ácaros administrada en pacientes con enfermedad alérgica demostró ser segura. No obstante, debe ser indicada y aplicada por personal clínico experto y advertírsele al paciente sobre los posibles riesgos y efectos adversos.

Palabras clave: inmunoterapia, alérgenos, hipersensibilidad.

Introduction: Allergic diseases affect the quality of life from who have them and their prevalence has significantly increased in recent years. Immunotherapy with allergenic extracts is used to improve the natural course of allergic diseases. However, it is still necessary to know their effectiveness and safety. Objective: To describe the systemic adverse reactions caused by the administration of subcutaneous immunotherapy with mites polymerized extracts. Materials and methods: It was performed a retrospective cohort study of patients diagnosed with allergic disease IgE mediated, who received subcutaneous immunotherapy with polymerized extracts of Dermatophagoides farinae, Dermatophagoides pteronyssinus $y$ Blomia tropicalis in the Clinical Allergy Service from IPS Universitaria of Universidad de Antioquia (Medellin, Colombia). A descriptive analysis of demographic and clinical variables and their association with the risk of systemic adverse reaction was made. Results: It was included a total of 622 patients; $92.8 \%$ were diagnosed with rhinitis, $60.3 \%$ with asthma, 56.9\% conjunctivitis and $19.6 \%$ atopic dermatitis. A total of $73(11.7 \%)$ patients experienced systemic reactions (ratio of reaction/injection $=1.06 \times 100$ injections). Of them, 47 (64.4\%) patients received intramuscular adrenaline. From all patients $93.1 \%$ had systemic reactions grade 1 and 2. Asthma was diagnosed with increased risk of systemic reaction (relative risk $=1.96)$. Conclusions: Subcutaneous immunotherapy with polymerised mites extracts administered in patients with allergic disease showed to be safe. However, it should be indicate and applied by trained clinical personnel and warning patients about the potential risks and adverse effects.

Key words: Immunotherapy, allergens, hypersensitivity.

Castelblanco-Arango IY, Gómez-Pineda PA, Cardona-Villa R. Determinación de la seguridad de la inmunoterapia subcutánea con extractos polimerizados de Dermatophagoides farinae, Dermatophagoides pteronyssinus y Blomia tropicalis administrada en pacientes con enfermedad alérgica. Medicina \& Laboratorio 2016; 22: 365-380. 
$\mathrm{L}$

a prevalencia de las enfermedades alérgicas tales como el asma, la rinitis, la

conjuntivitis y la dermatitis atópica han aumentado en los últimos años, convirtiéndose en un problema de salud pública en el mundo que afecta la calidad de vida y la funcionalidad de aquellos que las padecen [1]. En un estudio realizado entre los años 1998 y 2000 en seis ciudades principales de Colombia se reportó una prevalencia para asma, rinitis y dermatitis atópica del $22,7 \%$, el $31,3 \%$ y el $7,1 \%$, respectivamente [2], la cual se ha observado en incremento durante la última década [1].

En la actualidad se cuenta con múltiples tratamientos que intentan mejorar los síntomas y el curso clínico de las enfermedades alérgicas, entre estos se encuentran las terapias inmunomoduladoras como la inmunoterapia con extractos alergénicos que ayuda a controlar los síntomas, mejorar la calidad de vida, disminuir el uso de otros medicamentos, prevenir nuevas sensibilizaciones y modificar el curso clínico de las enfermedades alérgicas mediadas por IgE [3].

En estudios en los que han comparado los costos de los tratamientos convencionales con los de la inmunoterapia se ha demostrado un ahorro significativo del importe en el cuidado de la salud con el uso de esta última. Este efecto es incluso mayor cuando se considera el beneficio clínico persistente después de suspender el tratamiento, reflejado en la disminución de las consultas a urgencias, hospitalizaciones y medicamentos utilizados; además, de la prevención del ausentismo escolar y laboral que produce la reducción en los gastos para los pacientes y el sistema de salud [4].

Así mismo, múltiples ensayos clínicos han demostrado la efectividad de la inmunoterapia en pacientes con enfermedades alérgicas, siendo el único tratamiento que está dirigido a la causa subyacente [3]; incluso, se han logrado describir los beneficios clínicos a largo plazo después de recibir un curso completo durante tres a cinco años, los cuales parecen persistir hasta 12 años luego de ser suspendida $[5,6]$.

La inmunoterapia consiste en la administración repetida de alérgenos específicos a pacientes con una enfermedad alérgica mediada por anticuerpos tipo inmunoglobulina $\mathrm{E}(\mathrm{IgE})$, los cuales modulan el sistema inmune para que se generen células $\mathrm{T}$ reguladoras, se promueva un cambio del perfil Th2 al Th1 y se produzcan inmunoglobulinas G ( $\mathrm{IgG}$ ) [3], de manera que se alcance una tolerancia frente a los alérgenos a los cuales el paciente está sensibilizado y que son clínicamente relevantes [5].

Entre las vías de administración de la inmunoterapia se encuentran la subcutánea, la sublingual, la oral, la intrabronquial, la intranasal, la intralinfática y la epicutánea, aunque actualmente solo las dos primeras tienen registro sanitario en Colombia y han sido aprobadas para su uso en la práctica clínica [6]. Por otra parte, los regímenes para la administración de la inmunoterapia incluyen los lentos, los convencionales, los rápidos y los ultrarrápidos [7]. 
Las reacciones adversas a la inmunoterapia subcutánea son clasificadas en dos categorías: locales, que se manifiestan como eritema, prurito y edema en el sitio de la inyección, y sistémicas, que pueden variar en severidad de leve a muy graves, como la anafilaxia. La tasa de las reacciones adversas sistémicas por 100 inyecciones reportadas en la literatura en los últimos 15 años es de aproximadamente 0,2\% $(0,026 \%$ a $0,37 \%)$ en Estados Unidos y $0,01 \%$ a $0,3 \%$ en Europa [8].

En Colombia, el único estudio de seguridad de la inmunoterapia publicado hasta la fecha fue realizado en la ciudad de Medellín en el Servicio de Alergología de la IPS Universitaria de la Universidad de Antioquia, con extractos de ácaros despigmentados, polimerizados con glutaraldehído y adsorbidos en hidróxido de aluminio a una concentración de $100 \mathrm{DPP} / \mathrm{mL}$ (1 DPP es el resultante de despigmentar y polimerizar 1 HEP (equivalente de histamina en pruebas de punción; del inglés, Histamine Equivalent Prick)) de Dermatophagoides farinae, Dermatophagoides pteronyssinus y Blomia tropicalis (Laboratorios LETI ${ }^{\circledR}$, Madrid, España), utilizando un esquema rápido. En este estudio se reportó una tasa de reacciones sistémicas del 1,3\%, que corresponde a 8 de 575 pacientes vacunados, con una razón de reacción sistémica/inyección de 0,1 por 100 inyecciones [9].

Actualmente, existe un sistema de clasificación de las reacciones sistémicas de la inmunoterapia subcutánea propuesta por la Organización Mundial de Alergias (WAO; del inglés, World Allergy Organization) que comprende cinco grados según el compromiso de los órganos y que puede ser utilizado tanto en la práctica clínica como en la investigación (véase tabla 1) [8].

Dada la mayor relevancia de la inmunoterapia en el tratamiento de pacientes con enfermedad alérgica mediada por anticuerpos IgE es importante conocer su seguridad mediante la evaluación de las reacciones adversas sistémicas que puedan presentarse, ya que solo se puede justificar el uso de una terapia cuando ofrece un mayor beneficio que posibles riesgos. El objetivo de esta investigación fue describir las reacciones adversas sistémicas producidas por la administración de la inmunoterapia subcutánea con extractos polimerizados de Dermatophagoides farinae, Dermatophagoides pteronyssinus y Blomia tropicalis, establecer su frecuencia y severidad, explorar la posible relación entre los aspectos clínicos y la presencia o no de una reacción sistémica y, por último, describir el manejo médico realizado posterior a su aparición.

\section{Materiales y métodos}

\section{Tipo y población de estudio}

Se realizó un estudio de cohorte retrospectivo de sujetos que asistieron al servicio de Alergología Clínica de la IPS Universitaria de la Universidad de Antioquia de Medellín, Colombia. Se constituyó una cohorte histórica de los sujetos que recibieron 


\begin{tabular}{|c|c|c|c|c|}
\hline Grado 1 & Grado 2 & Grado 3 & Grado 4 & Grado 5 \\
\hline $\begin{array}{l}\text { Síntomas o signos de } \\
\text { uno de los siguientes } \\
\text { órganos: }\end{array}$ & $\begin{array}{l}\text { Síntomas o signos de } \\
\text { más de un sistema de } \\
\text { los órganos del grado } \\
1 \text { o al menos uno de } \\
\text { los siguientes: }\end{array}$ & $\begin{array}{l}\text { Uno o más de los } \\
\text { siguientes síntomas } \\
\text { o signos: }\end{array}$ & $\begin{array}{l}\text { Uno o más de } \\
\text { los siguientes } \\
\text { síntomas: }\end{array}$ & $\begin{array}{l}\text { Muerte } \\
\text { del pa- } \\
\text { ciente }\end{array}$ \\
\hline $\begin{array}{l}\text { Cutáneo: } \\
\text { Prurito generalizado, } \\
\text { urticaria, enrojecimiento } \\
\text { o calor } \\
\text { o } \\
\text { Angioedema (no laríngeo, } \\
\text { lengua o úvula) }\end{array}$ & $\begin{array}{l}\text { Respiratorio inferior: } \\
\text { Asma: tos, sibilan- } \\
\text { cias, disnea, dificultad } \\
\text { para respirar ( } p \text {. ej. } \\
\text { caída entre el } 20 \% \text { y } \\
\text { el } 40 \% \text { PEF }^{*} \text { o FEV1** } \\
\text { que responde a bron- } \\
\text { codilatador inhalado) }\end{array}$ & 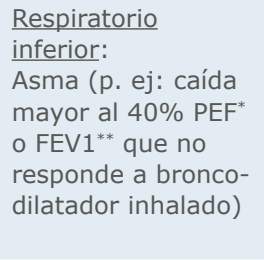 & $\begin{array}{l}\text { Respiratorio } \\
\text { inferior o superior: } \\
\text { Falla respiratoria } \\
\text { con o sin pérdida } \\
\text { de conciencia }\end{array}$ & \\
\hline $\begin{array}{l}\text { Respiratorio } \\
\text { superior: } \\
\text { Rinitis (p. ej. estornudos, } \\
\text { rinorrea, prurito nasal o } \\
\text { congestión nasal) } \\
\text { o } \\
\text { Aclaramiento faríngeo } \\
\text { (prurito en garganta) } \\
\text { o } \\
\text { Tos percibida de origen } \\
\text { en la vía aérea superior } \\
\text { (no de pulmón, laringe } \\
\text { o tráquea) }\end{array}$ & $\begin{array}{l}\text { Gastrointestinal: } \\
\text { Dolor abdominal, } \\
\text { vómito o diarrea }\end{array}$ & $\begin{array}{l}\text { Respiratorio } \\
\text { superior: } \\
\text { Edema laringe, } \\
\text { úvula o lengua con } \\
\text { o sin estridor }\end{array}$ & $\begin{array}{l}\text { Cardiovascular: } \\
\text { Hipotensión con } \\
\text { o sin pérdida de } \\
\text { conciencia }\end{array}$ & \\
\hline
\end{tabular}

\section{Conjuntival: Eritema, \\ Otro: \\ prurito o lagrimeo Cólico uterino}

\section{Otros:}

Náuseas, sabor

metálico o cefalea

La puntuación incluye un sufijo que denota el tiempo y administración de adrenalina como consecuencia de la aparición de los síntomas o signos de la reacción sistémica: a) menor a 5 minutos, b) entre 5 y 10 minutos, c) entre 10 y 20 minutos, d) mayor a 20 minutos y z) no aplicación de adrenalina

* PEF: flujo espiratorio máximo (del inglés, Peak Expiratory Flow)

${ }^{* *}$ FEV1: volumen espiratorio forzado en 1 segundo (del inglés, Forced Expiratory Volume in 1 second)

Tomado de "Speaking the same language: The World Allergy Organization Subcutaneous Immunotherapy Systemic Reaction Grading System" por L. Cox y colaboradores, 2010, J Allergy Clin Immunol, 125, p. 571. Copyright (c) 2010 por American Academy of Allergy, Asthma \& Immunology [8].

inmunoterapia subcutánea con extractos alergénicos de ácaros polimerizados con glutaraldehído y adsorbidos en gel de hidróxido de aluminio a una concentración de $10.000 \mathrm{UT} / \mathrm{mL}$ (Unidades Terapéuticas, equivalentes a 10.000 unidades biológicas del extracto no modificado) de Dermatophagoides farinae, Dermatophagoides pteronyssinus y Blomia tropicalis (Laboratorio Inmunotek ${ }^{\circledR}$, Madrid, España), durante enero de 2010 y diciembre de 2015.

Todos los participantes tuvieron un diagnóstico confirmado de al menos una enfermedad alérgica por parte de un médico especialista en alergología quien basó su diagnóstico en los resultados positivos de las pruebas epicutáneas de alergia 
(Prick Test) o IgE sérica específica para los ácaros en tratamiento ( $\geq 0,35 \mathrm{KUI} / \mathrm{L}$ ) y la aplicación de los criterios diagnósticos aceptados internacionalmente para cada condición.

Se requirió que todo participante hubiese recibido la inmunoterapia como parte de su tratamiento médico convencional y no de forma experimental; además, para su aplicación se solicitó que firmaran un consentimiento informado de procedimiento y que aceptaran el registro de su información en una base secundaria del Servicio para el control de los procedimientos relacionados con la inmunoterapia. Aquellos pacientes que recibieron también inmunoterapia sublingual con ácaros, inmunoterapia en otra institución o tenían antecedente de reacción sistémica no identificada en el Servicio de este estudio fueron excluidos.

\section{Protocolo de inmunoterapia}

El protocolo de inmunoterapia que se lleva a cabo en el Servicio consiste en un esquema ultrarrápido: a) inicio de esquema de inmunoterapia: 0,2 y 0,3 mL subcutánea en ambos brazos con intervalo de 30 minutos entre ambas dosis y b) esquema de mantenimiento de inmunoterapia: 0,5 $\mathrm{mL}$ subcutánea cada 4 a 8 semanas aplicando $10.000 \mathrm{UT} / \mathrm{mL}$. Posterior a cada aplicación de la inmunoterapia los pacientes son observados mínimo durante 60 minutos si tienen diagnóstico de asma y 30 minutos el resto de pacientes.

El procedimiento es vigilado permanentemente por un médico especialista en alergología; en caso de que se presente una reacción adversa se clasifica como local o sistémica. Si es de tipo local se prescriben medidas de control sintomático tales como la aplicación de hielo en la zona y la toma de medicamentos antihistamínicos o analgésicos. En caso de que sea de tipo sistémico se procede a clasificar de acuerdo con el sistema propuesto por la Organización Mundial de Alergias, se administra adrenalina y se observa al paciente durante al menos cuatro horas.

\section{Adquisición y análisis de datos}

La información se obtuvo de los registros sistematizados (fuentes de datos secundarias) del servicio de Alergología Clínica de la IPS Universitaria de la Universidad de Antioquia, que atiende pacientes con enfermedades alérgicas del departamento de Antioquia en Colombia. A través de un formulario estandarizado de información aplicado por un médico alergólogo, o un médico residente del programa de alergología entrenado previamente, se recolectó la información demográfica (edad, sexo), clínica (diagnósticos, tratamiento actual) y de inmunoterapia (tiempo de inicio, extracto aplicado, lote de vacuna, número de dosis recibidas, tipo de reacciones presentadas, tiempo de observación y tratamientos concomitantes recibidos).

Los registros fueron sistematizados en una hoja de cálculo diseñada en el programa Microsoft Excel ${ }^{\circledR} 2010$ en su versión para Windows (Microsoft, Washington, 
Estados Unidos) y se revisó que no hubiera información sin diligenciar o inconsistencia en los registros; en cuyo caso se hacía una nueva revisión de la información consignada en la base de datos del servicio.

Los datos fueron analizados con el programa estadístico Stata MP versión 13.1 para Windows (StataCorp ${ }^{\circledR}$, Texas, Estados Unidos). Se realizó un análisis descriptivo de las variables demográficas cuantitativas de los participantes por medio de medidas de posición como la mediana, el cuartil inferior y el cuartil superior, y medidas de dispersión como el valor máximo, el valor mínimo y el rango intercuartílico. Para las variables clínicas de tipo cualitativo se utilizaron frecuencias absolutas y porcentuales con sus respectivos intervalos de confianza. Para determinar la seguridad de la intervención se calculó la proporción de incidencia de reacciones adversas sistémicas, con su intervalo de confianza al 95\%, y la razón de reacción sistémica/inyección.

El criterio de normalidad de las variables cuantitativas se estableció mediante la prueba de Shapiro-Wilk; posteriormente, se realizó un análisis exploratorio para determinar la asociación entre la edad y el riesgo de reacciones adversas sistémicas a través de la prueba $U$ de Mann-Whitney para la diferencia de medianas en muestras independientes. Se evaluó la asociación entre el sexo, tipo de extracto y diagnósticos con el riesgo de reacciones adversas sistémicas a través de una prueba de chi cuadrado de independencia con el cálculo de la razón de proporciones de incidencia, con su respectivo intervalo de confianza al 95\%.

Dado que estas pruebas de hipótesis eran de carácter exploratorio se realizaron con un nivel de significancia de 0,05 sin hacer corrección por múltiples comparaciones. Para controlar el efecto de aspectos clínicos y demográficos confusores respecto a la presencia de una reacción sistémica se aplicó un modelo lineal generalizado de regresión de Poisson con varianza robusta, que permitió el cálculo de la razón de proporciones de incidencia ajustada y su intervalo de confianza al 95\%.

\section{Resultados}

\section{Características de los pacientes}

Se incluyeron en el estudio un total de 622 participantes que recibieron inmunoterapia subcutánea en el Servicio de Alergología Clínica durante el periodo en investigación, de los cuales 350 eran hombres (56,3\%; intervalo de confianza del $95 \%$ : 52,4\% a 60,2\%). La población de estudio se encontraba en un rango de edad entre los 2 y los 69 años, con una mediana de 12 años o menos (rango intercuartílico $=7$ ).

El diagnóstico más frecuente fue el de rinitis alérgica $(92,8 \%)$, seguido por asma alérgica $(60,3 \%)$, conjuntivitis alérgica $(56,9 \%)$ y dermatitis atópica $(19,6 \%)$; 
algunos de los pacientes con más de un diagnóstico. La mayoría de los pacientes fueron vacunados con el extracto de Dermatophagoides pteronissynus $(98,1 \%)$, seguido por la aplicación de Dermatophagoides farinae $(96,3 \%)$ y, por último, de Blomia tropicalis $(22,8 \%)$; sin embargo, el $96,3 \%$ de ellos recibieron al menos dos extractos alergénicos de forma simultánea (véase tabla 2).

\section{Reacciones sistémicas}

Un total de 73 pacientes $(11,7 \%$; intervalo de confianza del 95\%: 9,2\% a $14,3 \%$ ) experimentaron reacciones sistémicas. Se registraron 6.842 inyecciones administradas de inmunoterapia subcutánea para una razón de reacción/inyección de 1,06 x 100 inyecciones, tomando todas las reacciones. En 29 pacientes se presentó reacción sistémica grado 1 , en 39 grado 2 y en 5 grado 3 . No se registraron reacciones

Tabla 2. Diagnóstico de enfermedades alérgicas y tipo de inmunoterapia aplicada en los pacientes estudiados $(n=622)$

Características Frecuencia Porcentaje Intervalo de (\%) confianza del 95 (\%)

Diagnósticos

Rinitis alérgica

577

92,8

90,7 a 94,8

Asma alérgica

375

60,3

56,4 a 64,1

Conjuntivitis

354

56,9

53,0 a 60,8

alérgica

Dermatitis $122 \quad 19,6 \quad 16,5$ a 22,7 atópica

Extracto alergénico recibido

$\begin{array}{lccc}\begin{array}{l}\text { Dermatopha- } \\ \text { goides pte- } \\ \text { ronyssinus }\end{array} & 610 & 98,1 & 97,0 \text { a } 99,2 \\ \begin{array}{l}\text { Dermatopha- } \\ \text { goides farinae }\end{array} & 599 & 96,3 & 94,8 \text { a } 97,8 \\ \begin{array}{l}\text { Blomia } \\ \text { tropicalis }\end{array} & 142 & 22,8 & 19,5 \text { a } 26,1\end{array}$

tropicalis

Aplicación de extractos combinados

\begin{tabular}{lccc} 
No & 23 & 3,7 & 2,4 a 5,5 \\
$\begin{array}{l}\text { Dos extractos } \\
\text { combinados }\end{array}$ & 468 & 75,2 & 71,8 a 78,6 \\
$\begin{array}{l}\text { Tres extractos } \\
\text { combinados }\end{array}$ & 131 & 21,1 & 17,9 a 24,3 \\
\hline
\end{tabular}
adversas sistémicas grado 4 o 5 .

Respecto a los diagnósticos se encontró que de los 73 pacientes que presentaron reacciones sistémicas $70(95,9 \%)$ tenían rinitis alérgica, $53(72,6 \%)$ asma alérgica, $50(68,5 \%)$ conjuntivitis alérgica y $13(17,8 \%)$ dermatitis atópica. Al analizar el tiempo al cual se presentaba la reacción sistémica posterior a la aplicación de la inmunoterapia 69 pacientes (94,5\%; intervalo de confianza del 95\%: 89,2\% a $99,8 \%)$ presentaron la reacción antes de una hora y los 4 restantes (5,5\%; intervalo de confianza del $95 \%: 1,5 \%$ a $13,4 \%$ ) reaccionaron después de una hora, todos estos con diagnóstico de asma. Entretanto, del total de pacientes con reacciones sistémicas 27 (37,0\%; intervalo de confianza del 95\%: 25,6\% a 48,3\%) presentaron la reacción en la primera dosis del tratamiento y los demás $46(63,0 \%$; intervalo de confianza del $95 \%$ : $51,0 \%$ a $74,0 \%$ ) con las dosis de mantenimiento.

El tratamiento más frecuentemente usado frente a las reacciones sistémicas fue la adrenalina y los $\beta 2$-agonistas de corta acción, seguidos de la administración de antihistamínicos, oxígeno y esteroides; sin embargo, su uso varió según el grado de severidad (véase figura 1). Respecto al tratamiento de las reacciones sistémicas se encontró que en 7 pacientes (15,9\%; intervalo de confianza del 95\%: 4,6\% a 27,2\%) 
con reacción grado 2 o 3 se realizó observación médica por un tiempo menor a 3 horas y en 5 sujetos (12,8\% intervalo de confianza del 95\%: 1,8\% a 23,8\%) de los que presentaron reacción grado 2 no recibieron adrenalina (véase tabla 3 ).

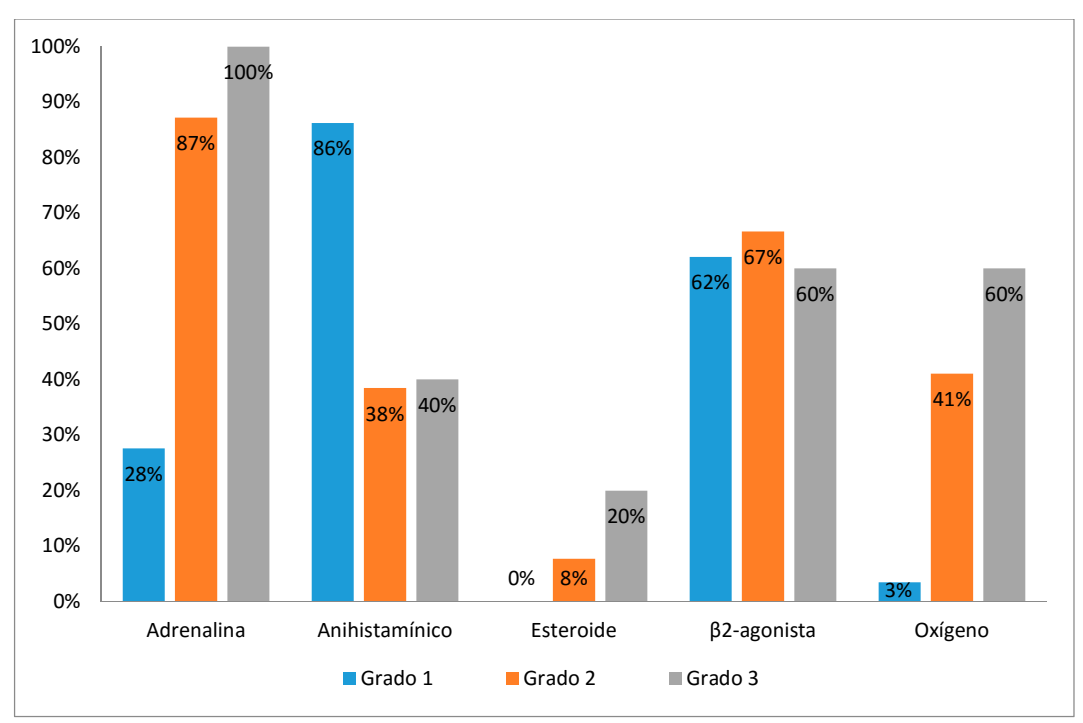

Figura 1. Porcentaje del uso de medicamentos según el grado de severidad de la reacción sistémica.

\begin{tabular}{|c|c|c|c|}
\hline Características & Frecuencia & Porcentaje (\%) & Intervalo de confianza del 95\% (\%) \\
\hline \multicolumn{4}{|l|}{ Sexo } \\
\hline Masculino & 29 & 39,7 & 28,2 a 51,2 \\
\hline Femenino & 44 & 60,3 & 48,1 a 71,5 \\
\hline \multicolumn{4}{|l|}{ Grado de severidad } \\
\hline Grado 1 & 29 & 39,7 & 28,2 a 51,2 \\
\hline Grado 2 & 39 & 53,4 & 41,7 a 65,1 \\
\hline Grado 3 & 5 & 6,8 & 1,0 a 12,8 \\
\hline Grado 4 & 0 & 0,0 & 0 \\
\hline Grado 5 & 0 & 0,0 & 0 \\
\hline \multicolumn{4}{|l|}{ Extracto vinculado a la reacción } \\
\hline Dermatophagoides farinae & 69 & 94,5 & 89,2 a 99,8 \\
\hline Dermatophagoides pteronyssinus & 68 & 93,2 & 87,2 a 99,1 \\
\hline Blomia tropicalis & 22 & 30,1 & 19,4 a 40,9 \\
\hline \multicolumn{4}{|l|}{ Tiempo de la reacción } \\
\hline Menos de 30 minutos & 34 & 46,6 & 34,9 a 58,3 \\
\hline
\end{tabular}




\begin{tabular}{lccc} 
Entre 30 y 60 minutos & 35 & 47,9 & 36,2 a 59,7 \\
Más de 60 minutos & 4 & 5,5 & 0,1 a 10,8 \\
\hline Tratamientos recibidos & 47 & 64,4 & 53,1 a 75,6 \\
\hline Adrenalina & 42 & 57,5 & 45,9 a 69,1 \\
Antihistamínicos & 20 & 27,4 & 16,9 a 37,9 \\
Oxígeno & 4 & 5,5 & 0,1 a 10,8 \\
Esteroides & & & 28,2 a 51,2 \\
\hline Tiempo de observación & 29 & 39,7 & 48,8 a 71,8 \\
\hline Menos de 4 horas & 44 & 60,3 & \\
Al menos 4 horas & &
\end{tabular}

\section{Factores de riesgo para las reacciones sistémicas}

Al realizar el análisis bivariado entre el riesgo de presentar una reacción sistémica y las variables en estudio se encontró un mayor riesgo en las mujeres, las personas con diagnóstico de asma y conjuntivitis y un menor riesgo en aquellos que habían sido vacunados con el extracto de Dermatophagoides pteronyssinus (véase tabla 4).

\begin{tabular}{|c|c|c|c|c|c|c|c|c|}
\hline \multirow[t]{2}{*}{ Factor de riesgo } & \multicolumn{2}{|c|}{$\begin{array}{l}\text { Reacción } \\
\text { sistémica }\end{array}$} & \multirow[t]{2}{*}{$\begin{array}{l}\text { Riesgo } \\
\text { relativo }\end{array}$} & \multirow{2}{*}{$\begin{array}{l}\text { Intervalo de } \\
\text { confianza } \\
\text { del } 95 \%(\%)\end{array}$} & \multirow[t]{2}{*}{$p$} & \multirow{2}{*}{$\begin{array}{l}\text { Riesgo } \\
\text { relativo } \\
\text { ajustado* }\end{array}$} & \multirow{2}{*}{$\begin{array}{l}\text { Intervalo de } \\
\text { confianza del } \\
95 \% \text { ajustado } \\
(\%)\end{array}$} & \multirow[t]{2}{*}{$p$ ajustado* } \\
\hline & Sí & No & & & & & & \\
\hline \multicolumn{9}{|l|}{ Sexo } \\
\hline Masculino & 44 & 228 & 1,95 & 1,26 a 3,04 & $<0,01$ & 2,12 & 1,37 a 3,29 & $<0,01$ \\
\hline Femenino & 29 & 321 & & & & & & \\
\hline \multicolumn{9}{|l|}{ Diagnóstico } \\
\hline \multicolumn{9}{|l|}{ Asma } \\
\hline Sí & 53 & 322 & 1,75 & 1,07 a 2,85 & 0,02 & 1,96 & 1,19 a 3,20 & $<0,01$ \\
\hline No & 20 & 227 & & & & & & \\
\hline \multicolumn{9}{|l|}{ Conjuntivitis } \\
\hline Sí & 50 & 304 & 1,65 & 1,03 a 2,63 & 0,03 & 1,57 & 0,98 a 2,50 & 0,06 \\
\hline No & 23 & 245 & & & & & & \\
\hline \multicolumn{9}{|l|}{ Rinitis } \\
\hline Sí & 70 & 507 & 1,82 & 0,60 a 5,55 & 0,27 & 1,63 & 0,51 a 5,22 & 0,41 \\
\hline No & 3 & 42 & & & & & & \\
\hline \multicolumn{9}{|l|}{ Dermatitis } \\
\hline Sí & 13 & 109 & 0,89 & 0,50 a 1,56 & 0,68 & 0,78 & 0,45 a 1,37 & 0,39 \\
\hline No & 60 & 440 & & & & & & \\
\hline
\end{tabular}




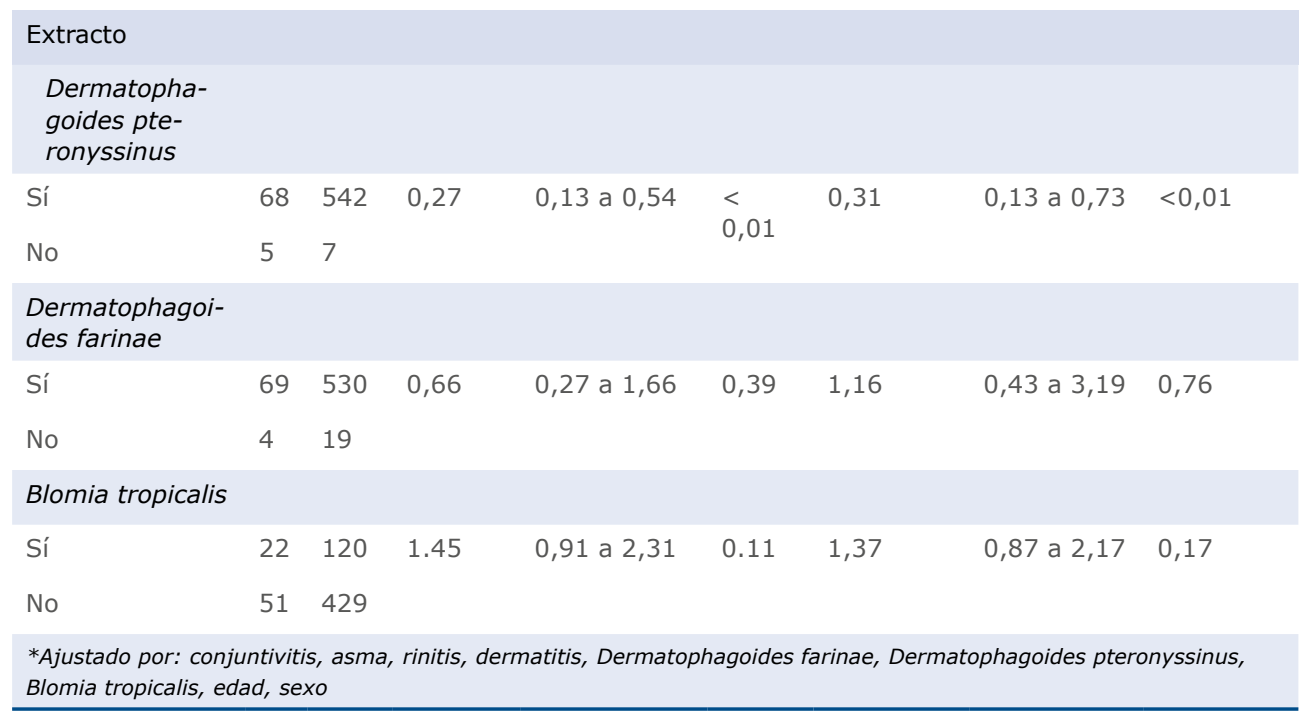

A partir de esta información se construyó un modelo multivariado con criterios clínicos y teóricos en el que se encontró que en las mujeres se incrementaba en 1,12 veces el riesgo de presentar una reacción sistémica; que quienes tenían un diagnóstico de asma alérgica presentaban un $96 \%$ más riesgo de reacción sistémica y que aquellos que fueron tratados con el extracto alergénico de Dermatophagoides pteronyssinus tenían un $69 \%$ menos riesgo de presentar reacción sistémica. A pesar de que la conjuntivitis había indicado ser un factor de riesgo en el análisis bivariado en el modelo multivariado no se preservó dicha asociación aunque se manifestó una tendencia a un mayor riesgo (véase tabla 4). No se halló asociación entre la edad y el riesgo de reacción sistémica $(p=0,83)$ ni un mayor riesgo en aquellos con diagnóstico de rinitis o dermatitis.

\section{Discusión}

Las reacciones sistémicas no fatales con la inmunoterapia subcutánea son infrecuentes y su reconocimiento y manejo oportuno hacen que sean generalmente leves y se resuelvan rapidamente. En el presente estudio la mayoría de las reacciones sistémicas fueron grado 1 y 2 , lo que resalta la ausencia de reacciones graves o fatales con esta intervención.

La razón de reacciones adversas sistémicas encontradas en este trabajo fue mayor que lo reportado en otros estudios, con 1,06 x 100 inyecciones frente a un promedio de 0,01 a 0,37 x 100 inyecciones reportado en los últimos 15 años en Europa y Estados Unidos [8]. Al comparar con los resultados reportados en el estudio realizado en Colombia con extractos de ácaros despigmentados, polimerizados de Dermatophagoides farinae, Dermatophagoides pteronyssinus y Blomia tropicalis del Laboratorios LETIß, entre 2007 y 2011, se encontró que la razón de 
reacciones adversas sistémicas fue también mayor $(1,06 \times 100$ inyecciones frente $0,1 \times 100$ inyecciones) [9].

Lo anterior podría ser debido a que previo al año 2010 se utilizaban diferentes sistemas de clasificación para categorizar las reacciones sistémicas con la inmunoterapia subcutánea, pero ninguna había sido aceptada a nivel mundial y solo hasta ese mismo año se publicó el consenso para la clasificación de las reacciones sistémicas [8]. De esta manera, los estudios realizados previamente pudieron no haber tenido en cuenta síntomas leves como prurito nasal, estornudos, obstrucción nasal, rinorrea, prurito ocular, epífora, hiperemia conjuntival, aclaramiento faringeo, tos de vía aérea superior y mareo, que actualmente están clasificadas dentro del sistema de puntuación como reacciones grado 1, lo que llevaría a un subregistro de las reacciones sistémicas. Es importante resaltar que el 39\% de las reacciones sistémicas observadas con la inmunoterapia en este trabajo fueron grado 1 y no se registraron reacciones grado 4 o 5 .

Otra posible explicación al incremento en la razón de reacciones adversas sistémicas presentadas en este estudio podría deberse a que los extractos de inmunoterapia utilizados tengan una mayor potencia o alergenicidad, expresada en las unidades biológicas o unidades terapéuticas por mililitro de la vacuna. No obstante, esto es difícil de determinar ya que no es posible hacer una comparación con el estudio previamente realizado en el servicio de Alergología Clínica de la Universidad de Antioquia, el cual utiliza unidades de medida diferentes que dependen del laboratorio que realizó la manufactura. Por tal razón, existe un interés creciente por realizar una estandarización de los extractos alergénicos utilizados actualmente para poder realizar estudios comparativos en cuanto a efectividad y seguridad [10].

Aunque las reacciones fatales por inmunoterapia son raras la tasa varía según la poblacion y el tipo de extracto. En 1987, Lockey y colaboradores [11] publicaron el reporte de la primera encuesta realizada a los miembros de la Academia Americana de Alergia, Asma e Inmunología (AAAAI; del inglés, American Academy of Allergy, Asthma \& Immunology) donde informaron 24 reacciones fatales entre 1959 hasta 1984. Posteriormente, en 1993, Reid y colaboradores [12] reportaron 15 muertes relacionadas con la inmunoterapia entre 1985 y 1989. En 2004, Bernstein y colaboradores [13] publicaron el resultado de una encuesta realizada a los médicos de la AAAAI entre 1990 y 2001, en la que se registraron 41 reacciones fatales con la inmunoterapia y 273 reacciones casi fatales, con un estimado de ocurrencia de reacciones fatales de 1 por cada 2.500 .000 de inyecciones y un promedio de 3,4 muertes por año. Finalmente, entre 2001 y 2007 se reportaron 6 muertes por inmunoterapia [14]; luego de esto no se han reportado nuevos casos de reacciones fatales.

En Europa, en una revisión realizada en Reino Unido entre 1957 y 1988 sobre la reacciones fatales con la inmunoterapia subcutánea, se identificaron 26 muertes, 
las cuales ocurrieron en los pacientes con diagnóstico de asma alérgica [15]. Esta mayor tasa de reacciones fatales reportadas en años previos podría ser explicada ya que anteriormente no existía regulación alguna de los extractos alergénicos; además, no se habían identificado los factores de riesgo para presentar reacciones sistémicas ni se habían realizado las modificaciones químicas y físicas actuales a los extractos alergénicos de inmunoterapia que aumentaran su seguridad [16]. En el presente el control de los extractos alergénicos está a cargo de la Agencia Europea de Medicamentos (EMA; del inglés, European Medicinal Agency) y la Administración de Alimentos y Medicamentos (FDA; del inglés, Food and Drug Administration) de los Estados Unidos [17].

Existen diferencias entre los extractos alergénicos utilizados en la inmunoterapia en países europeos y los Estados Unidos, la principal de ellas es la modificación química y física, además del uso de diferentes adyuvantes con fórmulas de liberación lenta que se realizan para disminuir su alergenicidad o producir agregados de alto peso molecular que mejoran la seguridad de los mismos. En Europa es más frecuente el uso de extractos modificados a diferencia de los Estados Unidos que utilizan principalmente extractos no modificados acuosos o glicerinados $[5,17]$. Los extractos alergénicos evaluados en este estudio son polimerizados con glutaraldehído y adsorbidos en gel de hidróxido de aluminio y producidos por el Laboratorio Inmunotek $®$ (Madrid, España). La polimerización reduce la capacidad de unión de los alérgenos a la IgE específica y mantiene la capacidad de reconocimiento por la IgG específica, lo que permite alcanzar la dosis de mantenimiento de forma rápida y segura [18].

Al analizar el tiempo en el cual se presentaba la reacción sistémica en los pacientes del presente trabajo, posterior a su aplicación, se encontró que fue durante la primera hora, lo cual es similar a lo reportado en estudios previos [15]; esto apoya la recomendación de observar a los pacientes por un periodo de $60 \mathrm{mi}$ nutos posterior a la aplicación de la inmunoterapia. En cuanto al manejo de las reacciones sistémicas la adrenalina fue administrada en un gran porcentaje de las reacciones grado 2 y en todas las reacciones grado 3, lo cual cumple con las recomendaciones de las guías internacionales $[5,8]$; aunque es de resaltar que cinco pacientes $(12,8 \%)$ de los que presentaron reacción grado 2 no recibieron adrenalina y el uso de la misma en las reacciones grado 1 se dejó a criterio médico.

El segundo medicamento más utilizado fue el $\beta 2$-agonista de corta acción en el $64,4 \%$ de los pacientes, posiblemente debido a un mayor compromiso del sistema respiratorio durante las reacciones sistémicas; no obstante, no es posible establecer la veracidad de esta afirmación ya que no se realizó un análisis detallado de los signos y síntomas que presentaron los pacientes incluidos en el estudio. Referente al tiempo de observación posterior a la reacción sistémica se encontró que en la mayoría de los pacientes la observación médica se realizó por un tiempo 
adecuado, el cual por consenso del grupo de alergólogos del Servicio de Alergología Clínica de la Universidad de Antioquia se había establecido, previamente, por tres a cuatro horas.

En la literatura se han reportado algunos factores de riesgo para presentar reacciones sistémicas durante el tratamiento con la inmunoterapia. En el primer estudio publicado en 1987 por Lockey y colaboradores [11] se encontraron como factores de riesgo los errores de dosificación, el uso concomitante de bloqueadores $\beta$, las reacciones sistémicas previas con la inmunoterapia y la administración durante la temporada pico de exposición al alérgeno. Otros estudios han reportado como factores de riesgo el asma no controlada, las reacciones sistémicas previas, el uso concomitante de bloqueadores $\beta$, la administración de la inyección en instituciones no especializadas, las comorbilidades como enfermedad cardiaca o pulmonar y el embarazo [16].

En este estudio se observó una posible relación entre el riesgo de reacción sistémica adversa y el sexo femenino y el diagnóstico de asma alérgica. El diagnóstico de conjuntivitis y el extracto alergénico de Blomia tropicalis presentaron una tendencia como factor de riesgo, sin ser estadísticamente significativa. No se encontró una posible asociación entre la edad y el riesgo de reacciones sistémicas ni un mayor riesgo en aquellos con diagnóstico de rinitis o dermatitis; además, no se evaluó el nivel de control de asma como factor de riesgo para presentar la reacción sistémica.

\section{Conclusiones}

Con los resultados observados en este estudio es importante tener en cuenta que para reducir la frecuencia de las reacciones adversas sistémicas por la inmunoterapia subcutánea es necesario cumplir ciertas condiciones, como una selección adecuada de los pacientes candidatos al tratamiento, la atención especial a los pacientes asmáticos que asegure la adherencia tanto a la inmunoterapia como al tratamiento convencional, la administración de la inmunoterapia solo en pacientes con control de la enfermedad alérgica de base, especialmente en los pacientes con diagnóstico de asma y, de ser necesario, el aplazamiento en la aplicación hasta lograr un buen control.

Además, se deben tener en cuenta otros aspectos como administrar la dosis correcta, evaluar la condición médica del paciente al momento de administrar la inmunoterapia, conocer los signos y síntomas de las reacciones sistémicas para hacer un reconocimiento temprano y poder dar un tratamiento oportuno, considerar descontinuar la terapia cuando se presentan múltiples reacciones sistémicas con la inmunoterapia, mantener al paciente en observación médica después de la aplicación de la inmunoterapia y garantizar una adecuada monitorización de los signos vitales en aquellos pacientes que hayan presentado una reacción sistémica. 
Aún no está claro si el tipo de extracto utilizado puede aumentar el riesgo de las reacciones sistémicas adversas, ya que como se mencionó anteriormente los diferentes extractos alergénicos disponibles en el mercado se expresan en diferentes unidades según el laboratorio que los manufactura, debido a que no hay una estandarización entre los extractos europeos de los diferentes laboratorios.

Hasta nuestro conocimiento no existen estudios, diferente al de Cardona y colaboradores (2014) [9], descrito previamente, en los que se evalúe la seguridad de la inmunoterapia subcutánea en Colombia; y en el mundo no se encontraron reportes de la frecuencia de reacciones sistémicas con los extractos utilizados en este estudio.

Los resultados obtenidos en el presente trabajo ratifican la importancia de conocer no solo la efectividad de los tratamientos disponibles, sino también su seguridad para así poder contar con toda la información acerca de los riesgos y beneficios de cada una de las opciones terapéuticas y poder brindar una atención individualizada del paciente alérgico.

\section{Limitaciones y fortalezas del estudio}

En el presente estudio el sesgo más frecuente pudo ser el de la información, el cual implica tener pérdidas de datos, subregistros o falta en la calidad de la información. En este caso, la presencia o no de las reacciones sistémicas secundarias a la inmunoterapia subcutánea podría no haberse consignado de forma detallada o suficiente respecto a las características y manejo de las mismas a través del tiempo, de acuerdo a la exposición de interés.

Como fortalezas del estudio se destaca que la mayoría de los pacientes que asistieron al Servicio de Alergología Clínica de la IPS Universitaria de la Universidad de Antioquia para la aplicación de la inmunoterapia subcutánea con ácaros fueron incluidos en el estudio lo que, si se considera que este es un centro de referencia para este tipo de terapias, va a permitir captar una adecuada muestra de usuarios. Además, todos los pacientes tenían el diagnóstico confirmado por un médico especialista en alergología de las enfermedades, incluidas las de etiología alérgica.

La inmunoterapia subcutánea con extractos polimerizados de Dermatophagoides farinae, Dermatophagoides pteronyssinus y Blomia tropicalis administrada en pacientes con asma, rinitis, conjuntivitis y dermatitis de etiología alérgica mostró 1,06 reacciones sistémicas por cada 100 inmunoterapias administradas, de las cuales el $93,2 \%$ fueron reacciones grado 1 y 2 ; por lo tanto, se considera una terapia segura con bajo riesgo de complicaciones o reacciones sistémicas graves. Por su parte, el asma fue el diagnóstico con el mayor riesgo de presentar una reacción sistémica (riesgo relativo $=1,96$ ), lo que indica que la inmunoterapia debe ser una terapia que se realice bajo supervisión clínica, que requiere que su 
indicación y aplicación sea por personal experto y se debe advertir a los usuarios sobre los posibles riesgos y efectos adversos.

\section{Agradecimientos}

Queremos dar nuestros más sinceros agradecimientos a Víctor Daniel Calvo Betancur, Magíster en epidemiología, por sus valiosos aportes en la elaboración del presente trabajo.

\section{Bibliografía}

1. Armentia A, Hernández N, Castrodeza J, Vaquero JL, López-Encinar $\mathbf{P}$, Rodrigo $\mathbf{P}$. Epidemiología de las enfermedades alérgicas. En: Peláez-Hernández A, Dávila-González IJ, eds. Tratado de alergología (ed 1a). Madrid, España: Ergon; 2007: 67-80.

2. Dennis R, Caraballo L, Garcia E, Caballero A, Aristizabal G, Cordoba $\mathbf{H}$, et al. Asthma and other allergic conditions in Colombia: a study in 6 cities. Ann Allergy Asthma Immunol 2004; 93: 568-574.

3. Cox L, Compalati E, Kundig T, Larche M. New directions in immunotherapy. Curr Allergy Asthma Rep 2013; 13: 178-195.

4. Lockey RF, Hankin CS. Health economics of allergen-specific immunotherapy in the United States. J Allergy Clin Immunol 2011; 127: 3943.

5. Cox L, Nelson H, Lockey R, Calabria C, Chacko T, Finegold $\mathbf{I}$, et al. Allergen immunotherapy: a practice parameter third update. J Allergy Clin Immunol 2011; 127: S1-55.

6. Burks AW, Calderon MA, Casale T, Cox $L$, Demoly $\mathbf{P}$, Jutel $\mathbf{M}$, et al. Update on allergy immunotherapy: American Academy of Allergy, Asthma \& Immunology/European Academy of Allergy and Clinical Immunology/PRACTALL consensus report. J Allergy Clin Immunol 2013; 131: 1288-1296 e1283.

7. Casanovas $\mathbf{M}$, Martin $\mathbf{R}$, Jimenez $\mathbf{C}$, Caballero $\mathbf{R}$, Fernandez-Caldas E. Safety of an ultra-rush immunotherapy build-up schedule with therapeutic vaccines containing depigmented and polymerized allergen extracts. Int Arch Allergy Immunol 2006; 139: 153-158.

8. Cox L, Larenas-Linnemann D, Lockey RF, Passalacqua G. Speaking the same language: The World Allergy Organization Subcutaneous Immunotherapy Systemic Reaction Grading System. J Allergy Clin Immunol 2010; 125: 569-574, 574 e561-574 e567.

9. Cardona R, Lopez E, Beltran J, Sanchez J. Safety of immunotherapy in patients with rhinitis, asthma or atopic dermatitis using an ultra-rush buildup. A retrospective study. Allergol Immunopathol (Madr) 2014; 42: 90-95.

10. Lowenstein $\mathbf{H}$. Characterization and standardization of allergen extracts. Chem Immunol Allergy 2014; 100: 323-332.

11. Lockey RF, Benedict LM, Turkeltaub PC, Bukantz SC. Fatalities from immunotherapy (IT) and skin testing (ST). J Allergy Clin Immunol 1987; 79: 660-677.

12. Reid MJ, Lockey RF, Turkeltaub PC, PlattsMills TA. Survey of fatalities from skin testing and immunotherapy 1985-1989. J Allergy Clin Immunol 1993; 92: 6-15.

13. Bernstein DI, Wanner $\mathbf{M}$, Borish $\mathbf{L}$, Liss GM. Twelve-year survey of fatal reactions to allergen injections and skin testing: 19902001. J Allergy Clin Immunol 2004; 113: 1129-1136.

14. Bernstein DI, Epstein T, Murphy-Berendts K, Liss GM. Surveillance of systemic reactions to subcutaneous immunotherapy injections: year 1 outcomes of the ACAAI and AAAAI collaborative study. Ann Allergy Asthma Immunol 2010; 104: 530-535.

15. Bernstein DI, Epstein T. Systemic reactions to subcutaneous allergen immunotherapy. Immunol Allergy Clin North Am 2011; 31: 241249, viii-ix.

16. Rank MA, Bernstein DI. Improving the safety of immunotherapy. J Allergy Clin Immunol Pract $2014 ; 2$ : 131-135.

17. Cox $\mathbf{L}$, Jacobsen $\mathbf{L}$. Comparison of allergen immunotherapy practice patterns in the United States and Europe. Ann Allergy Asthma Immunol 2009; 103: 451-459; quiz 459-461, 495.

18. Alvarez-Cuesta E, Aragoneses-Gilsanz E, Martin-Garcia C, Berges-Gimeno P, Gonzalez-Mancebo E, Cuesta-Herranz J. Immunotherapy with depigmented glutaraldehyde-polymerized extracts: changes in quality of life. Clin Exp Allergy 2005; 35: 572-578. 